\title{
Identification of genes and pathways related to lipopolysaccharide signaling in duckling spleens
}

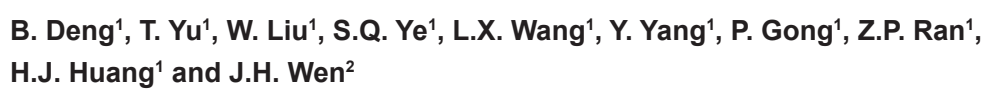

${ }^{1}$ Wuhan Institute of Animal Science and Veterinary Medicine, Wuhan Academy of Agricultural Science \& Technology, Wuhan, Hubei, China ${ }^{2}$ Wuhan University of Technology, Wuhan, China

Corresponding authors: B. Deng / J.H. Wen

E-mail: dengbing0906@163.com / wenjianghui86@163.com

Genet. Mol. Res. 14 (4): 17312-17321 (2015)

Received August 16, 2015

Accepted October 9, 2015

Published December 16, 2015

DOI http://dx.doi.org/10.4238/2015.December.16.32

ABSTRACT. Lipopolysaccharide (LPS), the major component of the outer cell wall of Gram-negative bacteria, activates the immune system and threatens the health of livestock and poultry. However, little is known about the genes and pathways involved in the immune response of ducklings to LPS. To elucidate the genes involved in the response of 7-day-old duckling spleens treated with LPS, RNA from LPS-treated and control duckling spleens was analyzed by RNA-Seq. The results showed 11,095 and 10,840 genes with >10 clean reads in the LPS-treated and control groups, respectively. Among these genes, 89 were differentially expressed $\left(\log _{2}\right.$ ratio $\geq 1, P \leq 0.01$, false discovery rate $\leq 0.001$ ); 67 of these were upregulated and 22 were downregulated in the LPS-treated group compared to the control. GO and GO-rich analysis showed that differentially expressed genes were enriched in 13 functional categories $(P<0.05)$. Pathway analysis and pathway richness analysis showed that differentially expressed genes were enriched in six pathway categories $(P<0.05)$. Further analysis showed that some immune system-related 
signaling pathways, such as the hematopoietic cell lineage, Toll-like receptor signaling pathway, $\mathrm{T}$ cell receptor signaling pathway, $\mathrm{T}$ cell receptor signaling pathway, complement and coagulation cascades, antigen processing and presentation, and chemokine signaling pathway, are active during the immune response. To confirm the RNA-Seq results, we detected CCL4, LBP, CD71, and STEAP3 expression using real-time $\mathrm{PCR}$ analysis, and the results were consistent with the RNA-Seq results. Our results provide new information on the genes involved in the immune response of duckling spleens to LPS.

Key words: Duck; Spleen; LPS; Differentially expressed genes

\section{INTRODUCTION}

Gram-negative bacteria (which appear red on Gram staining) can be detrimental to animal health in the livestock and poultry production industries. Lipopolysaccharide (LPS) is the major component of the outer cell wall of Gram-negative bacteria (Mani et al., 2012). Researchers have shown that a potent immunostimulant is responsible for much of the pathogenicity associated with Gram-negative bacterial infection (Leveque et al., 2003). When bacteria are killed by disinfectants, the presence of endotoxin (LPS) that remains in the animal building or in the air could damage the immune system and threaten the health of livestock and poultry (Pomorska et al., 2007).

LPS-induced damage has been studied in other species such as pig, rat, and chicken (Lipton et al., 2001; Parmentier et al., 2009; Chen et al., 2013); however, little information is known about the damage caused by LPS in ducks. Maloney and Gray (1998) showed that LPS could cause a temperature increase and prolonged fever in ducks. In animal production, the ducklings had a weaker defense and immune response to Gram-negative bacterial diseases compared with the adult ducks. Our research has also shown that the liver and spleen can be damaged by LPS in ducklings (unpublished data). Studies investigating the gene interactions involved in the immune response of duckling spleens to LPS will be useful for researchers and duck producers. However, there is little information about target genes and signaling pathways that are related to LPS exposure in ducklings.

Previous researchers have indicated that the TLR4 pathway plays an important role in pathogen recognition and activation of the innate immune system (Jault et al., 2004). In this pathway, LPS from Gram-negative bacteria is recognized by LPS-binding protein (LBP) and Toll-like receptor (TLR4), which together form a LPS-LBP-TLR4 receptor complex that activates signaling cascades via the nuclear factor-kappa B (NFkB), mitogen-activated protein kinase (MAPK), and interferon regulatory factor (IRF) pathways (Pålsson-McDermott and O'Neill, 2004; Newton and Dixit, 2012). In a study of chickens, researchers found that TLR4 plays an important role in the susceptibility of chickens to systemic salmonellosis (Leveque et al., 2003). Michailidis (2011) also found that chicken TLRs (cTLRs) respond to LPS and Salmonella enteritidis (SE).

Zhao (2013) found that the TLR4 gene has two alternative splice forms in duck: TLR4-a and TLR4-b, and both splice forms of TLR4 can be activated by LPS. The authors concluded that the TLR4 signaling pathway is very important for LPS recognition in ducks. However, the role of other genes and pathways that are involved in the immune response of ducklings to LPS remains unknown. Transcription-wide expression analyses could help to determine the key genes 
and pathways that play an important role in the immune response of ducklings following treatment with LPS. In this study, a transcriptome-wide expression analysis was performed using RNA-Seq to identify differentially expressed genes and pathways activated in ducklings treated with LPS. A large number of genes and pathways were revealed to be differentially expressed between the LPS-treated and control groups. Our analysis found that the differentially expressed genes and pathways might play an important role in the immune response of ducklings treated with LPS.

\section{MATERIAL AND METHODS}

\section{Experimental animals}

Ducks, which were provided by Wuhan Tianlv Technological Agriculture Company (P. R. China), were injected with $1000 \mu \mathrm{g} L S / \mathrm{kg}$ body weight at 7 -days of age $(\mathrm{N}=6)$ in the treatment group, and with the same volume of physiological saline in the control group. Twelve-hours later, tissue samples were carefully removed from their spleens, frozen in liquid nitrogen, and stored at $-70^{\circ} \mathrm{C}$ until further use. All research involving animals was conducted according to the relevant regulations (No. 5 proclaim of the Standing Committee of Hubei People's Congress) and approved by the Standing Committee of Hubei People's Congress, and the ethics committee of the Institute of Animal Science and Veterinary Medicine of Wuhan, Wuhan Academy of Agricultural Science \& Technology, P.R. China.

\section{RNA isolation and reverse transcription}

Spleen samples were placed in 2-mL tubes containing $1 \mathrm{~mL}$ TRIzol reagent (Invitrogen, San Diego, CA). One ceramic bead was added immediately to each tube. The tubes were then ground for 30 s using EASY GRIND equipment. Total RNA was extracted using TRIzol according to the manufacturer instructions (Invitrogen, Carlsbad, CA, USA). RNA quality was examined using a ND-1000 Nano-Drop spectrophotometer (Thermo, USA) and by $1.5 \%$ agarose gel electrophoresis; gels were stained with $1 \mu \mathrm{g} / \mathrm{mL}$ ethidium bromide.

One microgram total RNA was treated with DNase I (Fermentas, Japan) to remove the contaminating genomic DNA and then reverse transcribed to cDNA using a reverse transcription kit (Fermentas, Japan) according to the manufacturer protocol. The cDNA stocks were stored at $-70^{\circ} \mathrm{C}$ until analysis. A fragment of $\beta$-actin that spans an intron sequence was used to verify the efficiency of the reverse transcription reaction and to exclude genomic DNA contamination. The primer sequences were as follows: forward primer, 5'-AACTGGGATGACATGGAGAAGA; and reverse primer, 5'-ATGGCTGGGGTGTTGAAGGT (Li et al., 2012).

\section{RNA-Seq, data mining, and gene ontology (GO) analysis}

Spleen RNA from six ducklings treated with LPS were pooled, as were the control samples. Then, Solexa sequencing of the pooled RNA from the LPS-treated samples and the control samples was conducted at Shanghai Hanyu Bio-Tech (Li et al., 2012; Jiang et al., 2013). The Anase Duck database (ftp://ftp.plantbiology.msu.edu/pub/data/Eukaryotic_Projects/o_sativa/annotation_dbs/pseudomolecules/version_6.1/all.dir/) was employed for sequence analysis. A rigorous algorithm was developed by Shanghai Hanyu Bio-Tech based on the methods of Mortazavi and 
Wang (2008), and this algorithm was used to identify those genes that were differentially expressed between the two groups. A false discovery rate (FDR) of $\leq 0.001$ was used to identify differentially expressed genes. The differentially expressed genes were analyzed using an MA-plot-based method with the random sampling model of DEGseq. Pathway and GO term analyses were performed using hypergeometric distribution statistics (Wang et al., 2010).

\section{Real-time PCR analysis}

Quantitative real-time PCR was performed on a LightCycler 480 real-time PCR thermal cycler instrument (Roche) using SYBR Green qPCR mix (Tubo, Toyota). The real-time PCR system consisted of $10 \mu \mathrm{L} 2$ X SYBR Green qPCR mix, $0.5 \mu \mathrm{L}$ cDNA, $8.9 \mu \mathrm{L}$ double distilled water, and $0.6 \mathrm{~mL}$ primer pairs ( $10 \mu \mathrm{M}$ forward and $10 \mu \mathrm{M}$ reverse) in a total volume of $20 \mu \mathrm{L}$. Negative controls were performed by replacing the cDNA with double distilled water. The PCR parameters were as follows: denaturation at $95^{\circ} \mathrm{C}$ for $2 \mathrm{~min}$, followed by 40 cycles of denaturation at $95^{\circ} \mathrm{C}$ for $20 \mathrm{~s}$, annealing at the appropriate $\mathrm{Tm}$ for $20 \mathrm{~s}$, and extension at $72^{\circ} \mathrm{C}$ for $20 \mathrm{~s}$. Fluorescence was measured using the melting curve program with a heating rate of $2.5^{\circ} \mathrm{C} / \mathrm{s}$ from $57^{\circ} \mathrm{C}$ to $95^{\circ} \mathrm{C}$. All samples were measured three times. The sequences of the quantitative real-time PCR primers were as follows: CCL4 forward primer, 5'-ACTCTGACCCACCAACCT and reverse primer, 5'-GATTTCACGTCCCTTCCT $\left(\mathrm{Tm}=54^{\circ} \mathrm{C}\right)$; STEAP3 forward primer, 5'-GTCAGTAACAACACCGAAATCA and reverse primer, 5'-TTACCATCCCTGGCACCT $\left(\mathrm{Tm}=54^{\circ} \mathrm{C}\right)$; CD71 forward primer, 5'-TACTTAGCGGATGGTGATG and reverse primer, 5'-TTCTTGTTTGCCCTTTGC $\left(\mathrm{Tm}=60^{\circ} \mathrm{C}\right) ; \angle B P$ forward primer, 5'-TTGCCACTGCCAAGTTCCT and reverse primer, 5'-AGCTGGCGGTGTTGAAGAA $($ Tm = $55^{\circ} \mathrm{C}$ ); $\beta$-actin was used as the reference gene to normalize the quantification of each target mRNA. Primer sequences for $\beta$-actin were as follows: forward primer, 5'-AACTGGGATGACATGGAGAAGA and reverse primer, 5'-ATGGCTGGGGTGTTGAAGGT $\left(\mathrm{Tm}=60^{\circ} \mathrm{C}\right)$ ( $\mathrm{Li}$ et al., 2012). The level of relative mRNA expression of the target genes was calculated as fold changes of the threshold cycle $(\mathrm{Ct})$ value relative to the reference gene using the $2^{-\Delta \Delta C t}$ method. The $\mathrm{Ct}$ values of all samples were determined from the mean $\mathrm{Ct}$ calculated from the triplicate reactions; thus, $\Delta \mathrm{Ct}=\mathrm{Ct}$ (target gene) - Ct (reference gene). Then, the samples were normalized to the calibrator: $\Delta \Delta \mathrm{Ct}=\Delta \mathrm{Ct}$ (target gene) - $\Delta \mathrm{Ct}$ (calibrator). The calibrator was an untreated control or a particular stage of development to allow for the comparisons of the results (Livak and Schmittgen, 2001). In the present study, one of the control samples was chosen as the calibrator sample to evaluate the putative differential mRNA expression of the target genes. All the data were analyzed using SPSS software 13.0 for Windows (SPSS Inc. Chicago, IL). Data are presented as means \pm SE. Differences were considered significant if $\mathrm{P}<0.05$.

\section{RESULTS AND DISCUSSION}

\section{Overview of RNA-seq data}

To maximize the coverage of duck spleen mRNA by RNA sequencing, libraries were constructed by pooling RNA isolated from the spleens of six LPS-treated ducks to generate the treated library, and six pooled control samples to construct the control library. The RNA-Seq results yielded 7,106,149 (control library) and 7,627,610 (treated library) raw reads. Low quality reads [i.e., tags containing only adaptors and ambiguously called bases (reads that have many Ns)] were 
removed. In the control and treated groups, 6,922,339 and 7,401,114 clean reads, respectively, were obtained. The percentage of clean reads was $>97 \%$. A total of $5,396,114$ and $6,518,449$ reads were mapped in the control and treatment groups, respectively. Read analysis showed that $42.65 \%$ of the tags in the control library and $39.12 \%$ of the tags in the treated library were present in more than 100 reads. The number of genes with more than 10 clean reads was 10,840 and 11,095 , respectively. $A$ summary of the sequencing reads and matched genes is shown in Table 1. Information regarding the genes expressed in the control and treated libraries is shown in Table S1.

Table 1. A summary of the sequencing reads and matched genes.

\begin{tabular}{lcccc}
\hline \multirow{2}{*}{ Map to genome } & \multicolumn{2}{c}{ Control group } & \multicolumn{2}{c}{ LPS treatment group } \\
\cline { 2 - 4 } & Read number & Percentage (\%) & Read number & Percentage (\%) \\
\hline Clean reads & $6,922,339$ & 97.41 & $7,401,114$ & 97.03 \\
Total mapped reads & $5,396,114$ & 75.94 & $6,518,449$ & 85.46 \\
Total unmapped reads & $1,526,225$ & 21.48 & 882,665 & 11.57 \\
Unique match & $4,145,829$ & 58.34 & $4,847,888$ & 63.56 \\
Multi-position match & $1,250,285$ & 17.59 & $1,670,561$ & 3.90 \\
Perfect match & $2,120,821$ & 29.84 & $2,541,066$ & 33.31 \\
S5 bp mismatch & 611,489 & 8.61 & 787,768 & 10.33 \\
Genes with more than 100 reads & 5,888 & 42.65 & 5,363 & 39.12 \\
Genes with more than 10 clean reads & 10,840 & 78.51 & 11,095 & 80.93 \\
\hline
\end{tabular}

Reads: the original sequences sequenced by high-throughput sequencing technology (Sequenced Reads); Clean Reads: the reads that do not have joints or low quality reads; Total mapped reads: the total reads that can mapped to genes in the database; Total unmapped reads: the total reads that cannot be mapped to genes in the database; Unique match: reads can only mapped to one gene in the reference genome database; Multi-position match: reads can be mapped to multi-positions in the reference genome database; Perfect match: there is no mismatch when the reads are mapped to the genes in the reference genome database; $\leq 5 \mathrm{bp}$ mismatch: $\leq 5 \mathrm{bp}$ mismatch when the reads are mapped to the genes in the reference genome database.

\section{Differentially expressed genes in LPS-treated, 7-day-old ducks}

In this study, a rigorous algorithm was developed by Shanghai Hanyu Bio-Tech, based on the methods of Mortazavi et al. (2008) and Wang et al. (2010), to identify those genes that were differentially expressed between the two samples. These clean reads were mapped to 13,806 and 13,708 genes in the treatment and control groups, respectively. Among these genes, 89 were differentially expressed $\left(\log _{2}\right.$ ratio $\geq 1, P \leq 0.01$, FDR $\left.\leq 0.001\right)$. Compared to the treated library, the control library showed 67 upregulated and 22 downregulated genes according to the statistical criteria for raw reads and number of transcripts per million clean tags (TMP) (Table S2).

\section{Gene ontology analysis, gene ontology richness, pathway analysis, and pathway richness of differentially expressed genes}

The genes expressed in the spleens of 7-day-old ducks could be classified into 527 functions using Gene Ontology (GO) analysis (Table S3 $)$, and GO-rich analysis classified these genes into 58 functions (Table S4). Among the functions, the differentially expressed genes were enriched in 13 function categories $(P<0.05)$, including oxidoreductase activity, metabolism, cell differentiation, integrase activity, extracellular structure organization and biogenesis, extracellular space, enzyme regulator activity, extracellular matrix, antioxidant activity, cell death, pathogenesis, response to stimulus, and extracellular proteins (Table S4). 
Pathway analysis showed that the differentially expressed genes in the treated and control libraries could be grouped into 327 pathways (Table S5). Pathway richness analysis grouped the differentially expressed genes into 39 pathways. The differentially expressed genes in the same pathway are shown in Figure 1. Among the pathways, differentially expressed genes were enriched in six pathway categories $(P<0.05)$, which were as follows: signaling molecules and interaction, transport and catabolism, biosynthesis of other secondary metabolites, metabolism of cofactors and vitamins, immune system, and amino acid metabolism (Table S6).

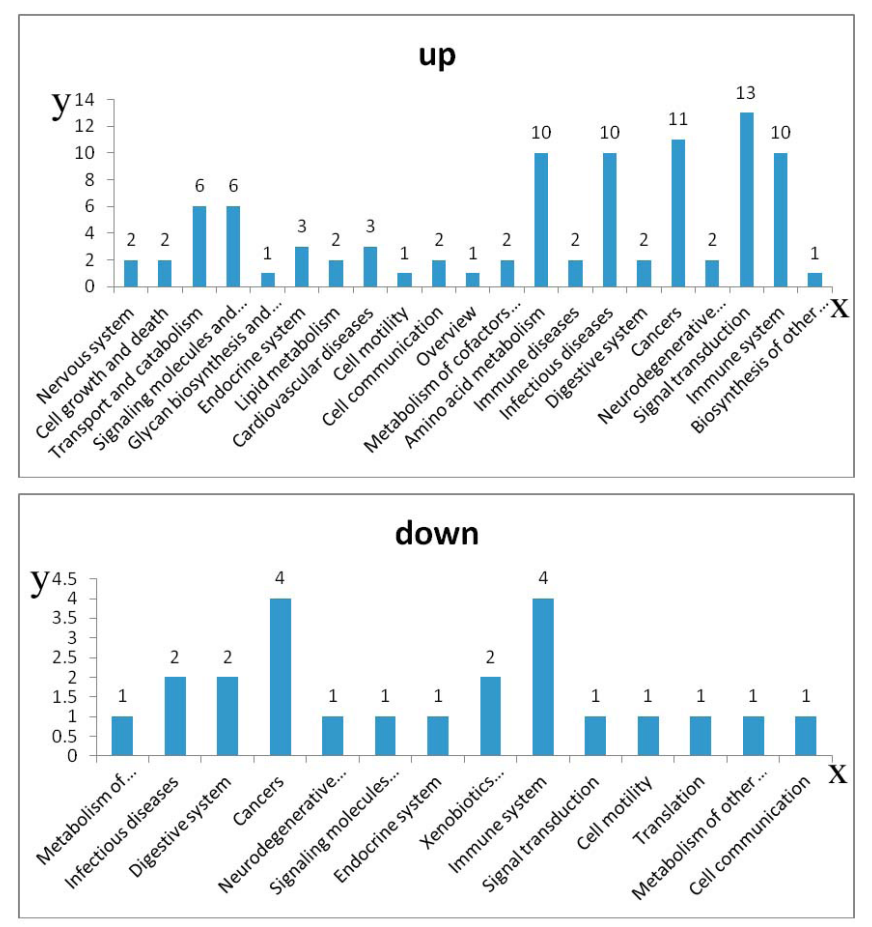

Figure 1. Number of differentially expressed genes in the same pathway (x-axis: pathway, $y$-axis: the number of differentially expressed genes).

Previous research has shown that TLR signaling pathways play an important role in pathogen recognition and activation of the innate immune system in mammals and chickens (Jault et al., 2004; Zhao et al., 2013). In this way, LPS from Gram-negative bacteria is detected by LBP and TLR4, which form an LPS-LBP-TLR4 complex and subsequently activates signaling cascades via the NF $\square$ B, MAPK, and IRF pathways (Pålsson-McDermott and O'Neill, 2004; Newton and Dixit, 2012). Further analysis revealed that some immune system-related signaling pathways are active during the response of ducks to LPS. Apart from TLRs, signaling pathways associated with the immune system include the hematopoietic cell lineage (MME [membrane metallo-endopeptidase] and CD71[TFRC, transferrin receptor]), T cell receptor signaling pathway (ITK [IL2-inducible T-cell kinase]), complement and coagulation cascades ( $C 1 R$ [complement component $1, r$ subcomponent] and $C 7$ [complement component 7]), antigen processing and presentation (CTSB [cathepsin B]), and chemokine signaling pathway (CCL4, CCLX [C-C motif chemokine], other), IL8RB (interleukin 8 receptor beta), and ITK (IL2-inducible T-cell kinase ). 
The hematopoietic cell lineage pathway is involved in the developmental progress of the lymphoid lineage of white blood cells or leukocytes, the natural killer (NK) cells and the T and B lymphocytes from hematopoietic stem cells (HSC) (Di Santo et al., 1999; Rothenberg and Taghon, 2005). The $\mathrm{T}$ cell receptor signaling pathway is mainly associated with T-cell proliferation, cytokine production, and differentiation into effector cells. The activation of $T$ lymphocytes is essential for an efficient immune response (Huang and Wange, 2004; Smith-Garvin et al., 2009). The activation of hematopoietic cell lineage and $\mathrm{T}$ cell receptor signaling pathways may indicate that $\mathrm{T}$ lymphocytes are proliferating and that cytokines are being produced in response to Gram-negative bacterial infection in ducklings.

The complement and coagulation cascades are key pathways in the complement system, which is a proteolytic cascade and a mediator of innate immunity, and a nonspecific defense mechanism against pathogens. The activation of this pathway leads to the opsonization of pathogens, recruitment of inflammatory and immunocompetent cells, and the direct killing of pathogens (Ruf et al., 2003; Turnberg and Botto, 2003). Based on our results, we hypothesize that the complement system participates in the immune response to Gram-negative bacterial infection in ducklings.

An inflammatory immune response is dependent on the recruitment of leukocytes to the site of inflammation following the detection of a foreign insult. During an inflammatory immune response, the chemokine signaling pathway is activated and transduced by chemokine receptors (G-protein coupled receptors) expressed on leukocytes. After chemokine receptor activation, the $\alpha-$ and $\beta y$-subunits of the $G$ protein dissociate to activate diverse downstream pathways that result in cellular polarization and actin reorganization (Wong and Fish, 2003; Thelen and Stein, 2008). The activation of chemokine signaling pathways suggests that chemokines are produced to provide directional cues for lineage determination in lymphocyte cells following the infection of ducklings with Gram-negative bacteria.

An overview of genes related to the immune system is shown in Table 2. LBP, IL8RB, CD71, C1R, C7, CTSB, CCLX, IL8RB, and CCL4 were upregulated in the treated library compared with the control library, while MME and ITK were downregulated (Table 2). As we know, ducklings have a weaker defense and immune response to Gram-negative bacterial diseases than adult ducks due to their immature immune systems. Our results indicate that the six signaling pathways could cooperatively participate in response to Gram-negative bacterial infection in ducklings. This information is useful for researchers and duck producers who aim to prevent Gram-negative bacteria prevention in duckling.

Table 2. A summary of the genes related to the immune system that were identified by RNA-Seq.

\begin{tabular}{|c|c|c|c|c|c|c|c|}
\hline \multirow[t]{2}{*}{ Genes } & \multicolumn{2}{|c|}{ Treatment } & \multicolumn{2}{|c|}{ Control } & \multicolumn{3}{|c|}{ Treatment/Control } \\
\hline & Read & RPM & Read & RPM & log2 (Treatment/Control) & q-value & Result \\
\hline$C D 71$ & 3287 & 981.329 & 1070 & 389.446 & 1.333 & 3.55E-174 & Up \\
\hline CCL4 & 630 & 188.086 & 106 & 38.581 & 2.285 & 4.04E-70 & Up \\
\hline C1R & 318 & 94.938 & 89 & 32.393 & 1.551 & $6.80 \mathrm{E}-21$ & Up \\
\hline$C 7$ & 498 & 148.677 & 120 & 43.676 & 1.767 & $1.52 \mathrm{E}-39$ & Up \\
\hline$\angle B P$ & 3593 & 1072.685 & 241 & 87.716 & 3.612 & 0 & Up \\
\hline CTSB & 5604 & 1673.066 & 1933 & 703.551 & 1.250 & $6.33 E-269$ & Up \\
\hline CCLX & 309 & 92.252 & 122 & 44.404 & 1.055 & $3.28 \mathrm{E}-11$ & Up \\
\hline IL8RB & 84 & 25.078 & 31 & 11.283 & 1.152 & 0.0006956 & Up \\
\hline MME & 508 & 151.663 & 994 & 361.785 & -1.254 & 4.86E-58 & Down \\
\hline ITK & 84 & 25.078 & 139 & 50.592 & -1.012 & $5.56 \mathrm{E}-06$ & Down \\
\hline
\end{tabular}

RPM $=$ reads per kilobases per million reads. $q$-value $=$ FDR (False discovery rate $)$ is used to determine the threshold $P$ value in multiple tests and analyses by manipulating the FDR value. q-value $<0.001$ means the differentially expressed genes were significantly different (Benjamini et al., 2001). 


\section{Real-time PCR confirmation of differential gene expression in ducks}

To confirm the differential gene expression observed by RNA-Seq, we measured the expression of $L B P, C C L 4$, and CD71, which are involved in immune function, and the STEAP3 gene, which is involved in cell growth and death, in the spleens of LPS-treated ducks using quantitative real-time PCR analysis. Those genes were selected from differentially expressed genes as determined from the RNA-Seq data. The samples were treated in the same way as those used for RNA-Seq.

Results of real-time PCR analysis revealed that $\angle B P$ is highly expressed after LPS treatment in ducklings (Figure 2A). LBP is a crucial molecule involved in the innate immune response to a bacterial challenge (Jault et al., 2004; Barton and Kagan, 2009; Kawai and Akira, 2010; Ding et al., 2013, Hsu et al., 2013). These results indicate that the TLR signaling pathway participates in the immune response to Gram-negative bacterial infection in ducklings.
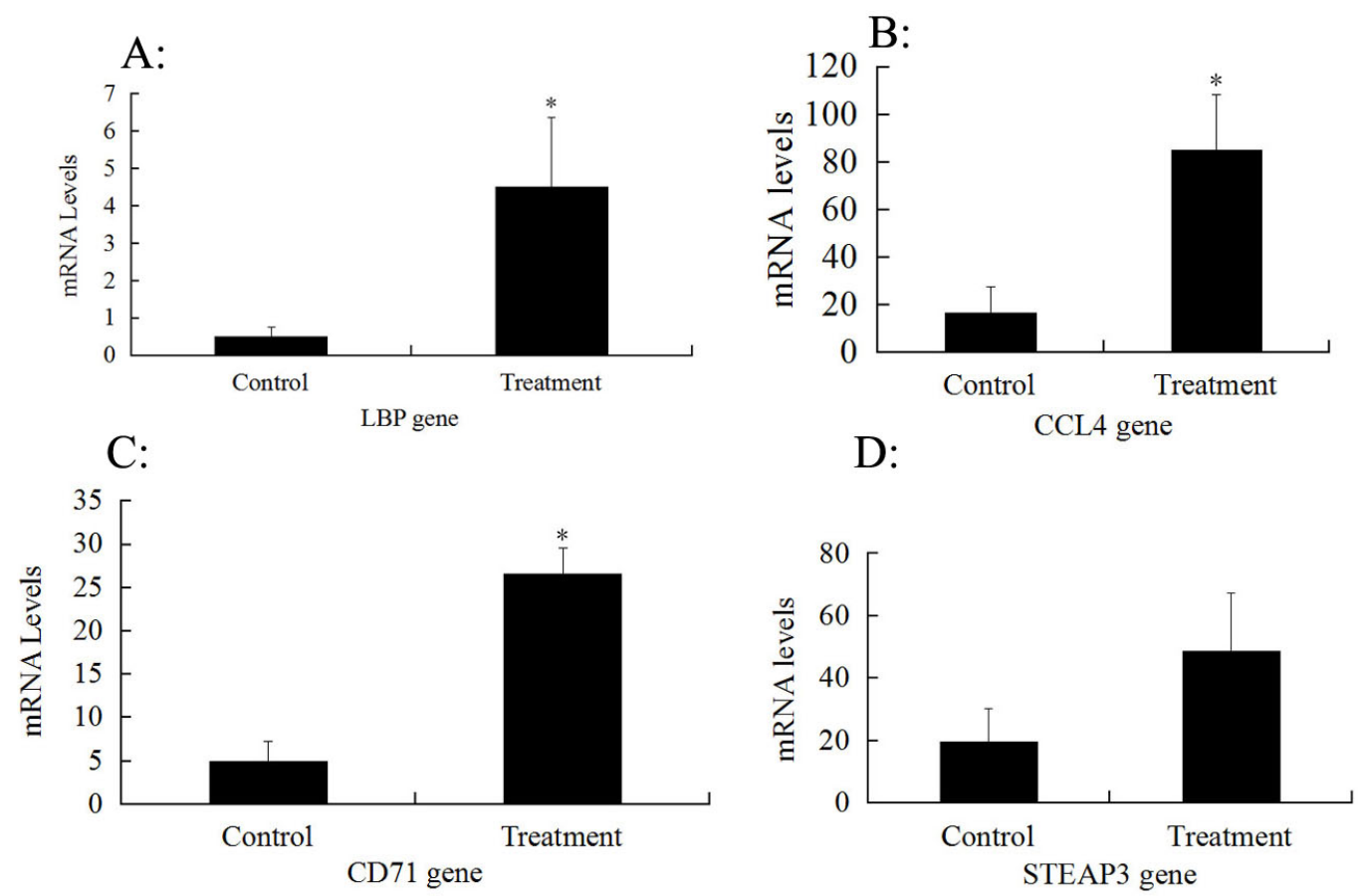

Figure 2. Confirmation of differential gene expression in control and LPS-treated animals. A. LBP mRNA expression was measured by real-time PCR analysis in control and LPS-treated animals. B. CCL4 mRNA expression was measured by real-time PCR analysis in control and LPS-treated animals. C. CD71 mRNA expression was measured by real-time PCR analysis in control and LPS-treated animals. D. STEAP3 mRNA expression was measured by realtime PCR analysis in control and LPS-treated animals. Asterisk denotes a significant difference $(P<0.05)$.

CC chemokine (motif) ligand 4 (CCL4) is indispensable for the chemoattraction of macrophages, natural killer cells, and lymphocytes in mammals. In LPS-stimulated fish, CCL4 expression did not increase until nearly $24 \mathrm{~h}$ after induction (Hsu et al., 2013). However, our results indicated that CCL4 was significantly upregulated in ducklings following treatment with LPS for $12 \mathrm{~h}$ (Figure 2B). This shows that this gene is involved in the inflammatory response in the spleens of ducklings. 
The CD71 (transferrin receptor) protein, which is closely associated with activation, proliferation, and differentiation, is expressed in liver cells, red blood cells, and lymphocytes along with many other cell surface proteins (Gutsol et al., 2013). The results show that CD71 gene expression was upregulated after treatment with LPS, which suggests that the function of $C D 71$ is associated with the proliferation of spleen lymphocytes (Figure $2 \mathrm{C}$ ).

The STEAP3 gene is part of the p53 signaling pathway and has a role in cell growth and death signaling, and is involved in the TLR4-mediated inflammatory response in macrophages (Zhang et al., 2012). Results from real-time PCR and RNA-Seq analysis showed that STEAP3 gene expression is upregulated after treatment with LPS, indicating that the product of this gene may be involved in the inflammatory response in duckling spleens (Figure 2D).

In this study, we performed transcription-wide expression analysis using RNA-Seq to identify genes that are differentially expressed in response to LPS treatment in ducks. Results indicated that 89 genes were differentially expressed; 67 of these genes were upregulated and 22 were downregulated during the immune response of ducklings to LPS. Our results also provide solid evidence for some of the functional mediators involved in mediating the response to LPS in ducklings, such as the hematopoietic cell lineage, TLR signaling pathways, T cell receptor signaling pathways, complement and coagulation cascades, antigen processing and presentation, and chemokine signaling pathways.

\section{Conflicts of interest}

The authors declare no conflict of interest.

\section{ACKNOWLEDGMENTS}

Research supported by the Elite Project of Wuhan Institute of Agricultural Science and Technology (\#YC201202), the earmarked fund for Modern Agri-industry Technology Research System (\#CARS-43-38), the Public science and technology research projects in Hubei Province (\#2012DBA48001), and the National Natural Science Foundation of China (\#31201766 and \#31201938).

\section{Supplementary material}

\section{REFERENCES}

Barton GM and Kagan JC (2009). A cell biological view of Toll-like receptor function: regulation through compartmentalization. Nat. Rev. Immunol. 9: 535-542.

Benjamini Y, Drai D, Elmer G, Kafkafi N, et al. (2001). Controlling the false discovery rate in behavior genetics research. Behav. Brain Res. 125: 279-284.

Chen P, Hou J, Ding D, Hua X, et al. (2013). Lipopolysaccharide-induced inflammation of bronchi and emphysematous changes of pulmonary parenchyma in miniature pigs (Sus scrofa domestica). Lab. Anim. 42: 86-91.

Di Santo JP, Aifantis I, Rosmaraki E, Garcia C, et al. (1999). The common cytokine receptor gamma chain and the pre-T cell receptor provide independent but critically overlapping signals in early alpha/beta T cell development. J. Exp. Med. 189: 563-574.

Ding PH, Wang CY, Darveau RP and Jin L (2013). Porphyromonas gingivalis LPS stimulates the expression of LPS-binding protein in human oral keratinocytes in vitro. Innate Immun. 19: 66-75.

Gutsol AA, Sokhonevich NA, Seledtsov VI, et al. (2013). Dexamethasone effects on activation and proliferation of immune memory T cells. Bull. Exp. Biol. Med. 155: 474-476. 
Hsu YJ, Hou CY, Lin SJ, Kuo WC, et al. (2013). The biofunction of orange-spotted grouper (Epinephelus coioides) CC chemokine ligand 4 (CCL4) in innate and adaptive immunity. Fish Shellfish Immunol. 35: 1891-1898.

Huang Y and Wange RL (2004). T cell receptor signaling: beyond complex complexes. J. Biol. Chem. 279: 28827-28830.

Jault C, Pichon L and Chluba J (2004). Toll-like receptor gene family and TIR-domain adapters in Danio rerio. Mol. Immunol. 40: 759-771.

Jiang S, Wei H, Song T, Yang Y, et al. (2013). Transcriptome comparison between porcine subcutaneous and intramuscular stromal vascular cells during adipogenic differentiation. PLoS One. 8: e77094.

Kawai T and Akira S (2010). The role of pattern-recognition receptors in innate immunity: update on Toll-like receptors. Nat. Immunol. 11: 373-384.

Leveque G, Forgetta V, Morroll S, Smith AL, et al. (2003). Allelic variation in TLR4 is linked to susceptibility to Salmonella enterica serovar Typhimurium infection in chickens. Infect. Immun. 71: 1116-1124

Li S, Wang C, Yu W, Zhao S, et al. (2012). Identification of genes related to white and black plumage formation by RNA-Seq from white and black feather bulbs in ducks. PLoS One 7: e36592.

Lipton BP, Bautista AP, Delcarpio JB and McDonough KH (2001). Effects of endotoxin on neutrophil-mediated I/R injury in isolated perfused rat hearts. Am. J. Physiol. Heart Circ. Physiol. 280: H802-811.

Livak KJ and Schmittgen TD (2001). Analysis of relative gene expression data using real-time quantitative PCR and the 2(-delta delta $\mathrm{C}(\mathrm{T})$ ) method. Methods 25: 402-408.

Maloney SK and Gray DA (1998). Characteristics of the febrile response in Pekin ducks. J. Comp. Physiol. B 168: $177-182$.

Mani V, Weber TE, Baumgard LH and Gabler NK (2012). Growth and development symposium: Endotoxin, inflammation, and intestinal function in livestock. J. Anim. Sci. 90: 1452-1465.

Michailidis G, Theodoridis A and Avdi M (2011). Effects of sexual maturation and Salmonella infection on the expression of Toll-like receptors in the chicken vagina. Anim. Reprod. Sci. 123: 234-241.

Mortazavi A, Williams BA, McCue K, Schaeffer L, et al. (2008). Mapping and quantifying mammalian transcriptomes by RNASeq. Nat. Methods 5: 621-628.

Newton K and Dixit VM (2012). Signaling in innate immunity and inflammation. Cold Spring Harb. Perspect. Biol. 4: pii: a006049

Pålsson-McDermott EM and O'Neill LA (2004). Signal transduction by the lipopolysaccharide receptor, Toll-like receptor-4. Immunology 113: 153-162.

Parmentier HK, Rodenburg TB, De Vries Reilingh G, Beerda B, et al. (2009). Does enhancement of specific immune responses predispose laying hens for feather pecking? Poult. Sci. 88: 536-542.

Pomorska D, Larsson L, Skorska C, Sitkowska J, et al. (2007). Levels of bacterial endotoxin in air of animal houses determined with the use of gas chromatography-mass spectrometry and Limulus test. Ann. Agric. Environ. Med. 14: $291-298$.

Rothenberg EV and Taghon T (2005). Molecular genetics of T cell development. Annu. Rev. Immunol. 23: 601-649.

Ruf W, Dorfleutner A and Riewald M (2003). Specificity of coagulation factor signaling. J. Thromb. Haemost. 1: 1495-1503.

Smith-Garvin JE, Koretzky GA and Jordan MS (2009). T cell activation. Annu. Rev. Immunol. 27: 591-619.

Thelen M and Stein JV (2008). How chemokines invite leukocytes to dance. Nat. Immunol. 9: 953-959.

Turnberg D and Botto M (2003). The regulation of the complement system: insights from genetically-engineered mice. Mol. Immunol. 40: 145-153.

Wang L, Feng Z, Wang X, Wang X, et al. (2010). DEGseq: an R package for identifying differentially expressed genes from RNA-seq data. Bioinformatics 26: 136-138.

Wong MM and Fish EN (2003). Chemokines: attractive mediators of the immune response. Semin. Immunol. 15: 5-14.

Zhang F, Tao Y, Zhang Z, Guo X, et al. (2012). Metalloreductase Steap3 coordinates the regulation of iron homeostasis and inflammatory responses. Haematologica 97: 1826-1835.

Zhao W, Huang Z, Chen Y, Zhang Y, et al. (2013). Molecular cloning and functional analysis of the duck TLR4 gene. Int. J. Mo. Sci. 14: 18615-18628. 